diseases which, with rising standards of hygiene and public services, are in themselves assuming milder forms (Ström, $1960,1967)$.

\section{Summary}

Among 516,276 triple-vaccinated children in Sweden from 1959 to 1965 neurological reactions to the vaccination occurred in 167 cases-destructive encephalopathy 3, convulsions 80, hypsarrhythmia 4, shock 54, uncontrollable screaming 24, serous meningitis 2 . Serous meningitis was also found in three out of nine examined cases of convulsions, and elevated protein in cerebrospinal fluid in one out of four examined cases of shock. Apart from these objective signs of meningeal involvement in certain cases, the study shows that in conjunction with both convulsions and shock there may be no or very little rise of temperature. The convulsive symptoms therefore cannot be classified as a matter of course as simple febrile convulsions.

The incidence of neurological reactions was $1: 3,600 \mathrm{vac}-$ cinated children $(1: 3,100$ if cases of persistent uncontrollable screaming are included), a rise in relation to the figure of $1: 6,000$ reported in a study from the years 1954 to 1958 . The rise is probably merely apparent, however, owing to the more watchful eye that is kept on these conditions. The more severe reactions leading to permanent injury seem to have decreased.

Analysis of the vaccinations of 208,186 children showed that $75.3 \%$ had been completely vaccinated. The figure for the non-vaccinated was $16.4 \%$, rather more than half of them due to the parents' refusal. Vaccination had not been completed in $8.3 \%$; in at least $3 \%$ the reason was probably the severe reaction to a preceding vaccination.

Apart from neurological reactions, erythema, exanthema, oedema, and gastrointestinal symptoms have also been reported. The great majority of reactions occurred after the first injection. Repeated injection appeared to produce the same reaction.

All forms of reaction can be explained as being of toxic origin. An allergic mechanism would appear to be of relatively limited significance ; a certain individual predisposition, however, seems to be a factor to reckon with.

Finally, it is emphasized that when nation-wide vaccination is recommended detailed information should be required concerning postvaccinal reactions, as is done in Sweden in conjunction with triple vaccination.

\section{REFERENCES}

Baird, H. W., and Borofsky, L. G. (1957). 7. Pediat., 50, 332.

Bower, B. D, and Jeavons, P. M. (1960), Brit. med. 7., 2,1453

Chao, D. H.-C., Taylor, F. M., and Druckman, R. (1957). \%. Pediat., 50,670 .

Ehrengut W. (1966). Impffibel, p. 244 . Stuttgart.

Hellström, B. (1962). Brit. med. $\dot{y}_{.,}, 2,1089$

Herrlich, A. (1965). Handbuch der Schutzimpfungen, p. 705. Berlin. Hopper, J. M. H. (1961). Med. Offr., 106, 241 .

Kringelbach, J., and Senstius, J. (1965). Nord. Med., 74, 1293.

Malmgren, B. Vahlquist, B., and Zetterström, R. (1960).' Brit. med. F., 2, 1800

Ström, J. (1960). Ibid., 2, 1184

Wilson, G. S. and Miles, A. A. (1964). Topley and Wilson's Principles of Bacteriology and Immunity, 5th ed., p. 2008. London.
Although much work has been done on clinical aspects of Burkitt's lymphoma, and intense interest has been shown in the relatively high rate of remissions and even "cures" among patients with this disease, there has been little analysis of longterm survival. The major reason for this has been the apparently enormous and seemingly insurmountable problems of keeping track of patients in the developing countries of Africa.

Clifford, working in Kenyatta National Hospital, Nairobi, Kenya, has been able to circumvent this problem to a very large extent by keeping patients in hospital for extended periods, and to date his series (Clifford, 1966 ; Pike, 1966) is the only major one in which complete survival information on the vast majority of patients is available.

Ideas on survival of patients attending Mulago Hospital, Kampala, Uganda, have been based on those known to have died plus those who continued to return to the follow-up clinic, but the fate of upwards of $50 \%$ of the patients leaving the hospital has not been known (Burkitt et al., 1965 ; Burkitt, 1966). However, by combining the data gathered by Mr. D. Burkitt and Mr. A. Lubega (the Kampala Cancer Registry

W.H.O. Senior Lecturer in Epidemiology, Department of Preventive Medicine, Makerere Medical School, P.O. Box 2072, Kampala, Uganda.

+ Member of the M.R.C. Statistical Research Unit, 115 Gower Street, London W.C.1, on secondment to the Department of Pathology, Makerere Medical School.

¥ Medical Assistant. Employed by Dr. Morrow under the U.S. National Cancer Institute (N.I.H.) Contran t No. PH-43-67-47.

An early draft of this paper was read at the East African Medical Research Council's Conference on "Cancer in Africa" which took place in Nairobi, Kenya, from 11-14 January 1967. social worker) with the data we have ourselves collected since November 1966 it has been possible to obtain survival information on 74 of the $80^{1}$ patients in a defined subpopulation of cases attending Mulago Hospital. This paper reports our findings in this group.

\section{Clinical Material}

The present study comprises the 80 patients diagnosed (microscopically, clinically, or at necropsy) as having Burkitt's lymphoma, who presented for the first time at Mulago Hospital between 1 January 1961 and 31 December 1965, and whose place of residence at onset date was in East or West Mengo Districts of Uganda (as demarcated on the last Ugandan Census in $1959^{2}$ ).

The East and West Mengo Districts, with a population of approximately $1 \frac{1}{2}$ million, comprise an area around Kampala with a radius of about $110 \mathrm{~km}$. (70 miles). To have extended the follow-up to include further districts of Uganda would have provided rapidly diminishing returns for the extra effort and expense involved.

'A table may be obtained from the authors giving for each of the 80 patients : reference number, age, sex, date of presentation at Mulago Hospital, length of history at that date, tumour involvement at presentation staging of the disease, if and when microscopically proved if and when if and when treatment stared and what it was (though not the dose as this was too often unknowable from the notes), date last seen, and condition, length of survival, dates of discharge "improved," dates of recurrences after discharge improved, sites of recurrences, and

therapy given.
${ }^{2}$ East African Statistical Department, Nairobi/Entebbe, 1960. 
We considered only patients presenting on or after 1 January 1961. It was about this time that "adequate" chemotherapy became available, and the criteria for diagnosis of the tumour had become relatively clear. The difficulties of tracing patients (and finding their notes) increase greatly with time after discharge, and though restricting ourselves in this way we still had a sufficient number of cases to make analysis instructive, while standing a good chance of finding the necessary high proportion of patients to make analysis valid. We did not consider patients presenting after 31 December 1965, because there had not been a sufficient lapse of time to make analysis of their survival very meaningful.

Of the 80 patients in the defined population at the beginning of this study (early November 1966) 36 had died in the hospital and another three were reliably reported to have died at home, while seven were known to be alive. Of the remaining 34, 11 had been unimproved by treatment and were thought to be dying on discharge, and 22 had been "successfully" treated but had not been seen since discharge. We were unable to find the hospital notes of the remaining patient, Case K229, though we were able to ascertain that she had been discharged. Thus of the $\mathbf{4 4}$ patients discharged from the hospital, the fate of 34 was in doubt. One of us (A.K.) devoted three months to tracing these patients. Four were found alive and free from tumour, ${ }^{3}$ the deaths of 22 patients were confirmed, and indirect evidence of the deaths of two was obtained. No information was forthcoming on the remaining six: three of these had been discharged improved, two had been discharged dying, and one was Case K229 mentioned above (this patient was not considered further here). The position is summarized in Table I.

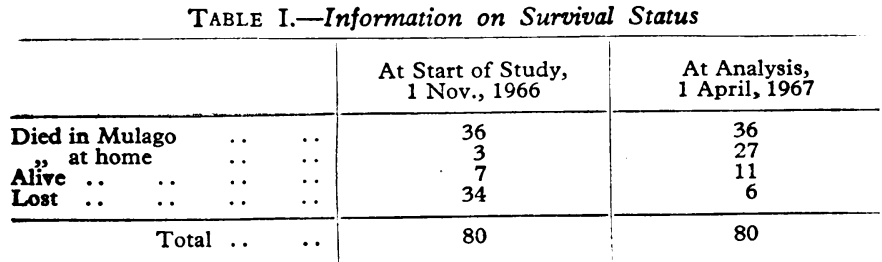

The diagnosis in 10 patients was made on clinical grounds alone: in two a clinical diagnosis was later confirmed at necropsy. Thus there was microscopical proof of diagnosis in 69 of the 79 patients. In all but one of these 10 not microscopically proved cases the patients are known to be dead. The other patient was improved by therapy but had extensive disease at discharge, and we have not been able to trace him. All "long-term" survivors had Burkitt's lymphoma diagnosed microscopically.

Of the 79 patients, 24 received no specific anticancer therapy. Seven of them were first diagnosed at necropsy ; 10 died before diagnosis was confirmed or treatment started ; five died during an operative or diagnostic procedure ; and two refused treatment-one with far-advanced disease was removed by his parents before biopsy or treatment and died at home the same day; the other (Case J152), a 9-year-old boy with all four quadrants of the jaw involved, was taken home by his parents after biopsy but before treatment. His parents stated that they then took him to the local traditional medical practitioner, after which he had a full remission, but the disease later recurred and he died 585 days after his initial presentation at Mulago. The remaining 55 patients had specific treatment for their disease.

All of these treated patients received some form of chemotherapy. The most frequently used first drug was cyclo-

- One of these patients (Case K76) was not actually seen, but the patient's father and the local chief stated that he was alive and well. The ar home was particularly disturbed during the "troubles" of 1966 and the patient was alleged to have been afraid to show himself owing to misgivings concerning the reasons for our visits (we attempted to see him on four different occasions). We have no (we attempted to see truth of these statements, and we have accepted them at their face value phosphamide, usually given intravenously but quite of ten orally and under varying dose schedules-most commonly a single dose, and the vast majority no more than two doses, of $40 \mathrm{mg} . / \mathrm{kg}$. body weight intravenously. Other drugs used were mercaptopurine, nitrogen mustard, methotrexate, and vincristine. These drugs were often given in combination, and for recurrences the drugs were usually given in turn if no response was being obtained. Seven patients had fairly extensive surgical excision of their tumour-five bilateral ovariectomies, one bilateral orchiectomy, and one abdominal mass removed.

\section{Survival Time}

We have counted survival time from the date of presentation at Mulago Hospital. This date was always easy to establish, and for the purpose of this paper may be regarded as that on which treatment began, a date that was not always completely clear from the hospital notes we managed to find.

For the purpose of the survival curve analysis the three "lost" patients who were discharged to die are assumed to have died on the day they left hospital, but for the two lost patients discharged improved we assume nothing further than that they were alive when they left hospital.

\section{Staging of the Extent of the Tumour}

Burkitt et al. (1965) have pointed out that the initial response of lymphoma cases in Mulago Hospital to chemotherapy was in part determined by the size of the tumour mass, and from a preliminary look at the survival data it was evident that the extent of tumour involvement was a critical factor in long-term prognosis. In our analysis we finally settled on the following crude division of cases into three categories, similar to those used in the staging of Hodgkin's disease:

Stage 1: Lymphoma limited to one anatomic region or to two contiguous anatomic regions on the same side of the diaphragm.

Stage 2: Lymphoma in two or more non-contiguous or more than two contiguous regions on the same side of the diaphragm.

Stage 3: Lymphoma involving any intrathoracic, intra-abdominal, or intracranial areas, or bone marrow, or more extensive involvement than stage 1 or stage 2 .

For example, involvement of both mandible and maxilla on one side of the face is classified as stage 2 , whereas involvement of the maxilla and orbit on one side is classified as stage 1 .

The major classification problem was whether liver or spleen enlargement constitutes tumour involvement in the absence of a laparotomy or needle biopsy-for this paper involvement was assumed only when there was a positive statement in the notes to this effect.

\section{Results}

Table II gives the survival times of the patients who received specific anticancer therapy. Fig. 1 shows the actuarially calculated survival curve for all these 55 cases. This curve

TABLE II.-Survival Times of Treated Patients in Days

\begin{tabular}{|c|c|c|c|}
\hline \multirow{2}{*}{$\begin{array}{c}\text { Age at } \\
\text { Presentation } \\
\text { in Years }\end{array}$} & \multicolumn{3}{|c|}{ Stage of Disease at Presentation } \\
\hline & 1 & 2 & 3 \\
\hline $0-4$ & $1505 t$ & $80,212,503 t, 943 t$ & $169 *, 1606 \dagger$ \\
\hline $5-9$ & $\begin{array}{c}13,1379 \dagger, 1389 \dagger, \\
1581 t, 1929 t\end{array}$ & $119,404^{*}$ & $\begin{array}{l}20^{*} \neq, 215,22,38,39 \neq, \\
425,47,500,80,107, \\
123,145,171,266, \\
320^{*}, 320,384^{*}, \\
479,804 \dagger\end{array}$ \\
\hline $10-14$ & & $31 \ddagger, 72$ & $\begin{array}{l}8,17,20^{*}, 87^{*}, 96, \\
102,147,159,263^{*}, \\
408,440\end{array}$ \\
\hline $\begin{array}{l}15-19 \\
20+\end{array}$ & & 227 & $\begin{array}{l}139,371,1848 t \\
44,67,228\end{array}$ \\
\hline
\end{tabular}

* Clinical diagnosis only. + Alive and clinically free of disease when last seen. $\ddagger$ Discharged "improved," not traced. \$Discharged unimproved, not traced. 
has the same general shape as the graph that was constructed from Clifford's data (Pike, 1966), with the characteristic, striking, long right-hand tail. Patients falling into this tail we regard as "long-term" survivors; all of them have been clinically free from disease for at least 496 days, while the longest remission in a patient who has subsequently died is 382 days-this is the patient who died 479 days after admission. The point on the " $\%$ survivors" axis at which the curve becomes horizontal we call " estimated long-term survival rate," and for the treated cases as a whole it is $21 \%$.

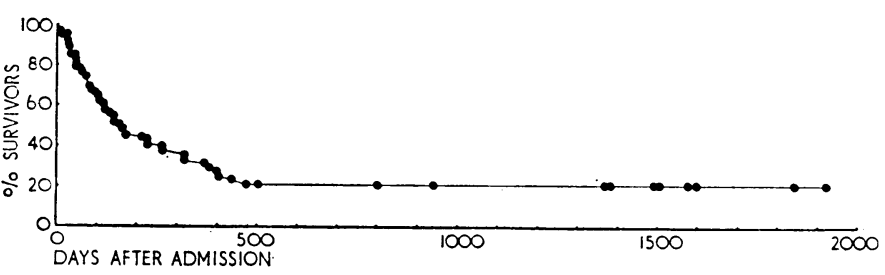

Fig. 1.-Survival curve for all 55 treated cases.

The most important determinant of survival in this series appears to be the extent of the disease on first admission (see Fig. 2 and Table III). The single stage 1 treated patient who has not survived was a 9-year-old girl who presented with a large tumour of the left thigh. This child died in the hospital two weeks after admission with an apparently fast-extending tumour, but it is not clear from the hospital notes why she died so soon and why necropsy was not performed. There is a suggestion on the histology request form that she might have had more extensive tumour involvement, and hence might not have been a stage 1 case.

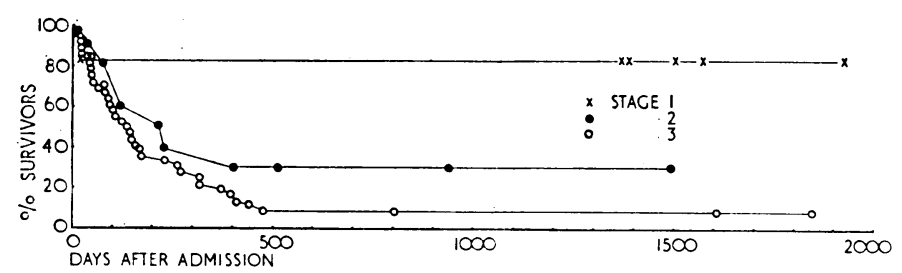

Fig. 2.-Survival times in Stages 1-3.

TABLE III.- " Long-term" Survival and Stage of Disease at Presentation

\begin{tabular}{c|c|c|c}
\hline Stage & $\begin{array}{c}\text { No. of at Risk } \\
\text { Patients* }\end{array}$ & $\begin{array}{c}\text { No. of Long-term } \\
\text { Survivors }\end{array}$ & $\begin{array}{c}\text { Estimated \% Long- } \\
\text { term Survivorst }\end{array}$ \\
\hline 1 & 6 & 5 & 83 \\
2 & $\mathbf{1 0}$ & 3 & 28 \\
\hline All patients & $\mathbf{3 4}$ & 3 & 8 \\
\hline
\end{tabular}

* Excludes "lost" patients.

† Actuarially calculated (see Figs. 1 and 2).

Younger patients did better than older patients (see Fig. 3). However, the age of the patient and the extent of his disease at presentation are highly interrelated (see Table II), and it is not possible with this limited number of cases to separate them completely satisfactorily. Fig. 4 shows that among stage 3 cases age is apparently irrelevant, though there is a hint of better prognosis for the younger patients with stage 2 disease. If attention is restricted to patients under 10 years of age it is clear from Table II that the stage of the disease remains of paramount importance.

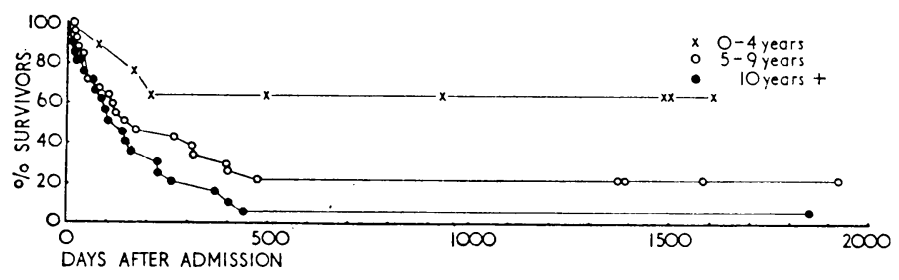

FIG. 3.-Survival times of patients according to age.
No relation exists-within a staging division-between length of history and prognosis.

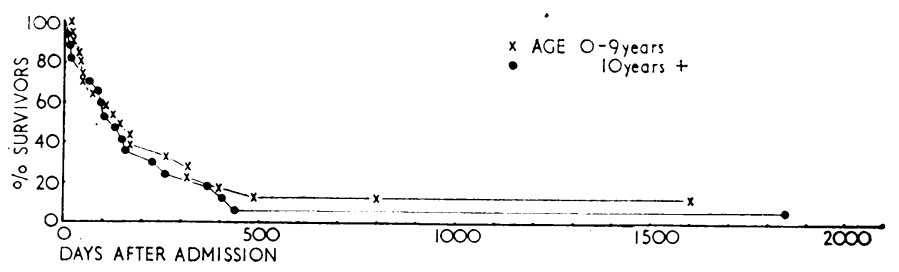

FIg. 4.-Survival times in stage 3 patients according to age.

It would clearly be extremely useful if on the basis of this series we could make some assessment of the relative efficacy of different chemotherapeutic regimens. However, as the extent of the disease at presentation appears to have a critical prognostic value, we need to compare different drugs (and different drug schedules) within a single staging category. Table IV shows that this is possible only with stage 3 cases; and Fig. 5 shows, within this staging division, the comparison of initial treatment with cyclophosphamide against all other drugs. For the first few months the patients receiving cyclophosphamide do better, but this advantage is not maintained.

TABLE IV.-Initial Chemotherapy

\begin{tabular}{c|c|c|c}
$\begin{array}{c}\text { Stage of } \\
\text { Disease at } \\
\text { Presentation }\end{array}$ & \multicolumn{2}{|c|}{ Drug } \\
\cline { 2 - 4 } & Cyclophosphamide & Other & Total \\
\hline 1 & 5 & 1 & 6 \\
2 & 10 & 1 & 11 \\
3 & 25 & 13 & 38 \\
\hline All patients & 40 & 15 & 55 \\
\hline
\end{tabular}

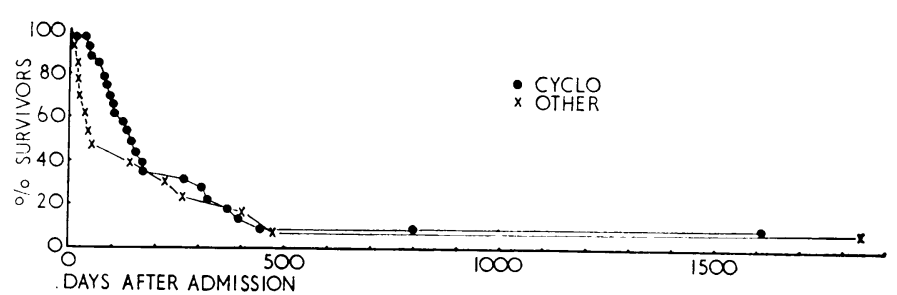

FIG. 5.-Survival times in stage 3 patients according to treatment.

\section{Long-term Survivors}

Ten of the 11 long-term survivors were under the age of 7 at presentation; while their sex ratio (seven males, four females) is identical to that of all treated cases.

Two important points arise from a study of the treatment of these 11 patients. The first is the apparent value of surgery in some cases. All three survivors with stage 3 disease had extensive surgical excision of their tumour-two had bilateral ovariectomies and one had a tumour mass that involved the bladder, right ureter, and ileum removed. Though there was possibly abdominal tumour remaining in all three patients, the bulk of it is excised. Two of these patients also had jaw or orbit involvement, but surgical excision was never attempted at these sites. An orchidectomy, first of the right testis and two and a half years later of the left, was performed on one stage 1 survivor (Case K99). Altogether seven patients had surgery with removal of most of the tumour mass, and four of these are long-term survivors.

The second point is that four of the survivors (all stage 1 cases) had recurrences. One of these patients was the testicular tumour case referred to in the previous paragraph. All of the remaining recurrences were in a site clearly different from the original one. These four patients all had complete clinical remissions followed by recurrences from 22 to 80 weeks later. Their recurrences were all still classifiable as stage 1 . 


\section{Discharged "Improved"}

To decide whether a patient has had a full or partial remission, or neither, is difficult in the best of circumstances. It is particularly so when these decisions have to be made retrospectively on the basis of case notes alone. We have nevertheless attempted this, and a certain amount of useful information was obtained.

Mulago Hospital notes invariably stated for each discharged patient whether " improved " or " not improved," and we have accepted this dichotomous definition of remission as the best we could manage-we have thus taken "improved" to mean "in remission" except when the notes also showed that the patient still had "significant" residual tumour (this was not always easy to decide).

Table $\mathrm{V}$ shows the discharged improved rate related to the extent of the disease at presentation-this relationship has been reported in a slightly different manner and in further detail as regards extent of remission by Burkitt et al. (1965).

TABLE V.-Dicharged "Improved" and Stage of Disease at Presentation

\begin{tabular}{c|c|c|c}
\hline Stage & $\begin{array}{c}\text { No. of } \\
\text { Patients }\end{array}$ & $\begin{array}{c}\text { No. Discharged } \\
\text { "Improved" }\end{array}$ & $\begin{array}{c}\text { \% Discharged } \\
\text { "Improved" }\end{array}$ \\
\hline 1 & 6 & 5 & 83 \\
2 & 31 & 8 & 73 \\
3 & 38 & 21 & 55 \\
\hline All patients & 55 & 34 & 62 \\
\hline
\end{tabular}

Of the 24 patients discharged improved who subsequently had recurrences 10 did not bother to return to Mulago Hospital -two of these were stage 2 and the others stage 3 cases. The length of time between the discharge of these patients and death are (in days): $51,63,88,124,133,162,240,285$, 307 , and 391 . It is surprising that these patients did not think it worth their while to return, in particular those with lengthy remissions.

\section{Discussion}

In this series the stage of the disease at presentation was of critical prognostic importance. However, as is well known to anyone working with this tumour, the disease does not invariably progress from stage 1 through stage 2 to stage 3 . In fact, many stage 3 patients present with abdominal lesions alone, and by our definition these cases have been stage 3 throughout the course of their disease. From the histories of the vast majority of the stage 3 cases with both abdominal and jaw lesions it was simply not possible to tell the time sequence of symptoms; and in the few instances where the jaw tumour appeared to have preceded the abdominal involvement one was always doubtful of the true significance of this, since the jaw lesions were so much more obvious. Similarly, though a number of stage 2 patients who later died had progressed to stage 3 before death, so that this sequence seems at least to be possible, we have to bear in mind that the first physical examination might well have missed a small abdominal mass. There are a number of cases in which the abdominal tumour appears to have preceded the jaw lesion.

Surgical reduction of abdominal tumour masses produced our only stage 3 survivors. This result, we feel, lends support to the finding that the critical factor in prognosis is the extent of disease at presentation. The risks attendant on abdominal surgery would therefore appear to be worth taking. This is in accordance with the results obtained in Ibadan, Nigeria (Ngu, 1964), where the only long-term survivors with stage 3 disease had excisions of their abdominal tumours.

Four of the five stage 1 long-term survivors had recurrences. Treatment of recurrences is thus distinctly worth while. In two of the stage 2 deaths the patients had had long remissions before dying at home without returning to Mulago Hospital, and it is possible that they might have survived if they had returned for further treatment at recurrence.

The results obtained from this series do not agree in all respects with the results found by Clifford (1966).

We divided Clifford's patients into our three staging categories on the basis of the details given in his paper, but both the "remission" rate (about $80 \%$ ) and the long-term survival rate (about $17 \%$ ) were independent of this division. A possible explanation of this discrepancy is to be found in the different drug dosages used in Nairobi and Kampala.

On average Clifford gives about two to three times the initial dose of drug given at Mulago Hospital. This appears to have resulted in a number of important differences in what we might term secondary response. A number of Clifford's stage 3 patients are long-term survivors on chemotherapy alone, a phenomenon not observed in our series. But the recurrence times for tumours of small extent are on the whole much shorter than those observed in Kampala.

There is some evidence, both clinical and experimental (Burchenal and Burkitt, 1967), that host defences may play an important role in survival in Burkitt's lymphoma. Regressions (one in this series, see above) and "cures" have been noted after little or no specific chemotherapy ; while Klein and his co-workers have obtained results interpreted as showing a tumour specific component in the sera of Burkitt's lymphoma patients in complete remission. If one adopts this proposition then one may argue that the chemotherapeutic agents used might not only affect tumour tissue but also affect the patients' immune response, and the balance between these two results of therapy might be critical. Clifford is giving a quantity of drug which will destroy the greater percentage of tumours (at least of large ones), but this results in a greater reduction in the patients' own defences-some of his cases die of leukopenia, and the initial drop in the Nairobi survival curve is very steep, so that the end-result is a similar "success" rate in Nairobi and Kampala, but with marked differences in effect of initial tumour location-these arguments in a slightly different form have been previously suggested by Burkitt et al. (1965).

This, however, is all very speculative, and the major problems of which drug to give, the best total dose (of a particular drug) to give, and whether this should depend on the "extent" of the disease, remain unresolved. It is our belief that these problems will not be resolved without strictly controlled clinical trials. Joint trials conducted simultaneously in a number of centres, with each of these centres drawing patients from an area as wide as possible, and the maintenance of a complete follow-up, would be of enormous value in getting sufficient numbers of cases together in a short time, and it is hoped that this can be arranged.

This study has shown that successful follow-up of a limited group of young patients, even retrospectively, is possible in Uganda. We attribute our success to having a non-hurried approach, in particular to being willing to spend considerable time explaining what we were doing to people encountered in our searches. After this explanation information was often forthcoming that the informant either denied knowing beforehand or did not bother to remember.

Note.-Mr. Clifford has now reviewed his data, employing a system of staging similar to that used here, and reports that he has found some relationship between this method of staging and prognosis. The relationship was not nearly as striking as that found in our series, and we still feel that the factors discussed above are those most likely to account for the difference.

\section{Summary}

Follow-up of 80 cases of Burkitt's lymphoma treated in Mulago Hospital, Uganda, has shown that the "long-term" 
survival rate is approximately 1 in 5 . This rate is markedly dependent on the extent of anatomical involvement of the lymphoma at presentation in this series, but in the previously reported series of Clifford (1966) this is not so. A possible reason for this discrepancy is sought in the different dosages of drugs used in the two centres.

This investigation represents a continuation and extension of the work initiated by Mr. D. Burkitt. We are grateful to Mr. Burkitt and Mr. S. Kyalwazi, who between them treated nearly all the cases reported in this series, for permission to follow up their patients. Special acknowledgement is due to both the past and the present pathologists at Makerere Medical School, and in particular to Dr. D. H. Wright, for carrying out the microscopy of the cases considered in this paper. We would like to thank Mrs.
Barbara Wright (registrar, B.E.C.C.-financed Kampala Cancer Registry) for the assistance she gave us in collecting details of the patients. We gratefully acknowledge the financial support of the U.S. National Cancer Institute (N.I.H.) under their Contract No. PH-43-67-47, and of the British Empire Cancer Campaign for Research.

\section{REFERENCRS}

Burchenal, J. H., and Burkitt, D. (Eds.). (1967). Treatment of Burkitt's Tumour. Heidelberg. (In press.)

Burkitt D. (1966). F. roy. Coll. Surg. Edinb., 11, 170.

Huth, M. S. R., and Wright, D. H. (1965). Cancer (Philad.), 18, 399.

Clifford, P. (1966). E. Afr. med. F., 43, 179.

Ngu, A. V. (1964). Brit. 尹. Cancer, 19, 101

Pike, M. C. (1966). Lancet, 2, 856.

\title{
Hypokalaemic Alkalosis and Hyperplasia of the Juxtaglomerular Apparatus without Hypertension or Oedema*
}

\author{
L. J. BEILIN, † M.D., M.R.C.P. ; N. SCHIFFMAN, $\ddagger$ M.D. ; M. CRANE,§ M.D. ; D. H. NELSON, $\|$
}

Hyperaldosteronism is commonly associated with either hypertension and hypokalaemic alkalosis as in renal vascular hypertension, malignant hypertension, and primary aldosteronism, or with oedema as in cirrhosis with ascites, the nephrotic syndrome, and chronic congestive heart failure. A dissociation between the hypertensive, potassium-losing, and continued sodium-retaining effects of aldosterone has previously been noted (Nelson and August, 1959) in patients who become oedematous. Three patients have been described with hypokalaemia, hyperaldosteronism, and hyperplasia of the juxtaglomerular apparatus but without hypertension or oedema (Bartter et al., 1962 ; Boucher et al., 1964 ; Ames et al., 1965). The present case, like the other similar cases, apparently represents another instance in which aldosterone in excess has not resulted in hypertension. The cause of the syndrome described in these cases is unknown, but it has been suggested that it represents an important aberration from the normal relations between the renin-angiotensin-aldosterone system and blood pressure control.

\section{Case Report}

The patient was an 18-year-old negro male whose illness dates from infancy. He was delivered after a seven-month pregnancy and was well until 4 months of age, when he stopped growing. At 7 months he was admitted to the Children's Medical Center in Dallas, Texas, after two weeks' incessant vomiting. He was found to be grossly dehydrated, wasted, and drowsy. He was in opisthotonos, but Trousseau and Chvostek signs were negative. The testes were undescended and he had a first-degree hypospadias. Investigations at that time revealed a serum bicarbonate of $46 \mathrm{mEq} /$ 1., serum chloride $74.6 \mathrm{mEq} / 1$., serum sodium $123 \mathrm{mEq} / \mathrm{l}$., serum potassium $4.8 \mathrm{mEq} / 1$, and a blood $p \mathrm{H}$ of 7.65 . Idiopathic alkalosis was diagnosed, and he was initially treated with ammonium

- From the University of Southern California School of Medicine and the Los Angeles County General Hospital, Los Angeles, California. This work was supported in part by a grant from the USPH Service, General Clinical Research Centre Grant FR-43.

t Holder of Sir Henry Wellcome Travelling Fellowship. Present address : Hammersmith Hospital, London W.12.

Instructor in Medicine, University of Southern California School of Medicine, Los Angeles.

5 National Institures of Health, Career Development Awardee-9-K3HE-7627, and Research Professor of Medicine, Loma Linda University, Loma Linda, California.

|| Investigator. Howard Hughes Medical Institute. Present address: University of Utah College of Medicine, Salt Lake City, Utah. chloride, sodium chloride, and deoxycortisone without effect. He eventually recovered after subcutaneous fluids and oral feeding, and was discharged clinically well but with persistent hypochloraemic alkalosis.

He grew slowly, walked at 15 months, but at 4 years was noted to have polyuria and polydipsia. He was then reinvestigated and found to have one testis in the scrotum and a bone age of $3 \frac{3}{4}$ years. Hypochloraemic alkalosis was present with serum chloride of $87 \mathrm{mEq} / 1$., serum bicarbonate $41 \mathrm{mEq} / 1$., a blood $\mathrm{pH}$ of 7.59, and a creatinine clearance of $116.6 \mathrm{ml} . / \mathrm{min}$. He was given no further treatment at that time.

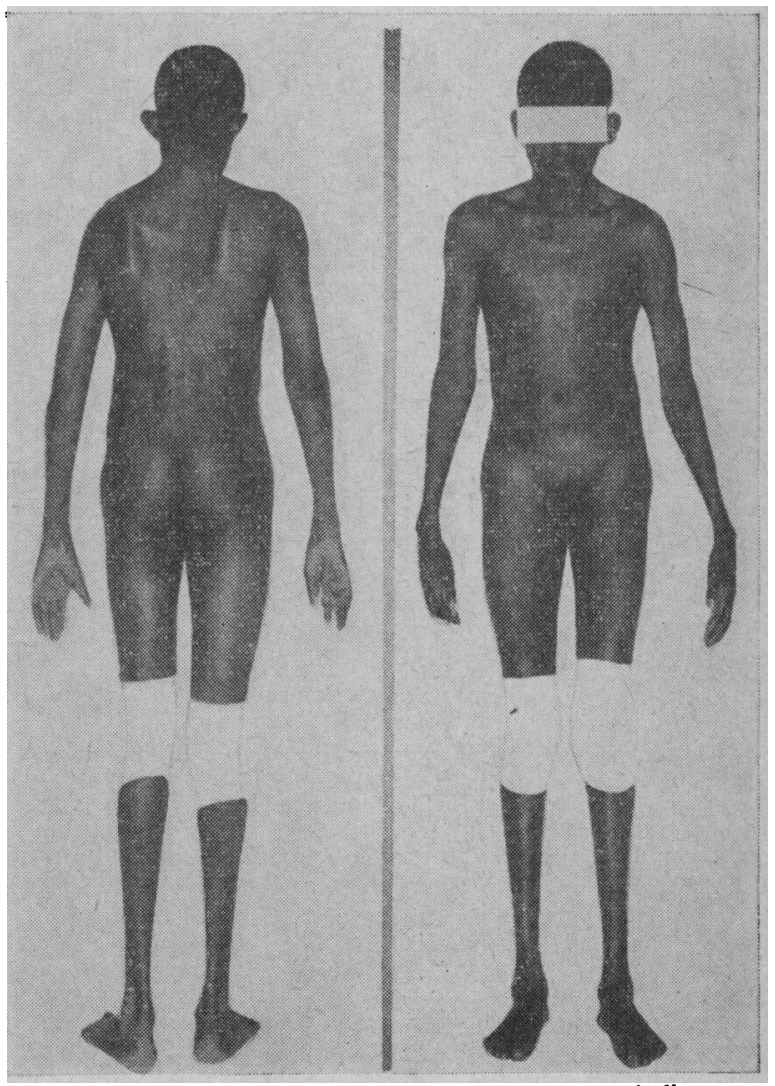

Fig. 1.-Patient aged 18, with prepubertal genitalia. 